\title{
Amplitude analysis of the decay $D^{0} \rightarrow K^{-} K^{+} \pi^{0}$
}

B. Aubert, ${ }^{1}$ M. Bona, ${ }^{1}$ D. Boutigny, ${ }^{1}$ Y. Karyotakis, ${ }^{1}$ J. P. Lees, ${ }^{1}$ V. Poireau,${ }^{1}$ X. Prudent, ${ }^{1}$ V. Tisserand,,${ }^{1}$ A. Zghiche, ${ }^{1}$ J. Garra Tico, ${ }^{2}$ E. Grauges, ${ }^{2}$ L. Lopez, ${ }^{3}$ A. Palano, ${ }^{3}$ G. Eigen, ${ }^{4}$ B. Stugu, ${ }^{4}$ L. Sun, ${ }^{4}$ G. S. Abrams ${ }^{5}$ M. Battaglia, ${ }^{5}$ D. N. Brown, ${ }^{5}$ J. Button-Shafer, ${ }^{5}$ R. N. Cahn, ${ }^{5}$ Y. Groysman, ${ }^{5}$ R. G. Jacobsen, ${ }^{5}$ J. A. Kadyk, ${ }^{5}$ L. T. Kerth, ${ }^{5}$ Yu. G. Kolomensky, ${ }^{5}$ G. Kukartsev, ${ }^{5}$ D. Lopes Pegna, ${ }^{5}$ G. Lynch, ${ }^{5}$ L. M. Mir, ${ }^{5}$ T. J. Orimoto, ${ }^{5}$ M. T. Ronan, ${ }^{5, *}$

K. Tackmann, ${ }^{5}$ W. A. Wenzel, ${ }^{5}$ P. del Amo Sanchez,${ }^{6}$ C. M. Hawkes, ${ }^{6}$ A. T. Watson, ${ }^{6}$ T. Held, ${ }^{7}$ H. Koch, ${ }^{7}$

B. Lewandowski, ${ }^{7}$ M. Pelizaeus, ${ }^{7}$ T. Schroeder, ${ }^{7}$ M. Steinke, ${ }^{7}$ D. Walker, ${ }^{8}$ D. J. Asgeirsson, ${ }^{9}$ T. Cuhadar-Donszelmann, ${ }^{9}$ B. G. Fulsom, ${ }^{9}$ C. Hearty, ${ }^{9}$ N. S. Knecht, ${ }^{9}$ T. S. Mattison, ${ }^{9}$ J. A. McKenna, ${ }^{9}$ A. Khan, ${ }^{10}$ M. Saleem, ${ }^{10}$ L. Teodorescu, ${ }^{10}$ V.E. Blinov ${ }^{11}$ A. D. Bukin, ${ }^{11}$ V. P. Druzhinin, ${ }^{11}$ V. B. Golubev,${ }^{11}$ A. P. Onuchin,,${ }^{11}$ S. I. Serednyakov, ${ }^{11}$ Yu. I. Skovpen, ${ }^{11}$ E. P. Solodov, ${ }^{11}$ K. Yu Todyshev, ${ }^{11}$ M. Bondioli, ${ }^{12}$ S. Curry, ${ }^{12}$ I. Eschrich, ${ }^{12}$ D. Kirkby, ${ }^{12}$ A. J. Lankford, ${ }^{12}$ P. Lund, ${ }^{12}$ M. Mandelkern, ${ }^{12}$ E. C. Martin, ${ }^{12}$ D. P. Stoker, ${ }^{12}$ S. Abachi, ${ }^{13}$ C. Buchanan, ${ }^{13}$ S. D. Foulkes, ${ }^{14}$ J. W. Gary, ${ }^{14}$ F. Liu, ${ }^{14}$

O. Long, ${ }^{14}$ B. C. Shen,${ }^{14}$ L. Zhang, ${ }^{14}$ H. P. Paar, ${ }^{15}$ S. Rahatlou, ${ }^{15}$ V. Sharma, ${ }^{15}$ J. W. Berryhill, ${ }^{16}$ C. Campagnari, ${ }^{16}$ A. Cunha, ${ }^{16}$ B. Dahmes, ${ }^{16}$ T. M. Hong, ${ }^{16}$ D. Kovalskyi, ${ }^{16}$ J. D. Richman, ${ }^{16}$ T. W. Beck, ${ }^{17}$ A. M. Eisner, ${ }^{17}$ C. J. Flacco, ${ }^{17}$ C. A. Heusch, ${ }^{17}$ J. Kroseberg, ${ }^{17}$ W. S. Lockman, ${ }^{17}$ T. Schalk, ${ }^{17}$ B. A. Schumm, ${ }^{17}$ A. Seiden, ${ }^{17}$ D. C. Williams, ${ }^{17}$ M. G. Wilson, ${ }^{17}$ L. O. Winstrom, ${ }^{17}$ E. Chen, ${ }^{18}$ C. H. Cheng, ${ }^{18}$ F. Fang, ${ }^{18}$ D. G. Hitlin,${ }^{18}$ I. Narsky, ${ }^{18}$ T. Piatenko, ${ }^{18}$ F. C. Porter ${ }^{18}$ G. Mancinelli, ${ }^{19}$ B. T. Meadows, ${ }^{19}$ K. Mishra, ${ }^{19}$ M. D. Sokoloff, ${ }^{19}$ F. Blanc, ${ }^{20}$ P. C. Bloom,${ }^{20}$ S. Chen, ${ }^{20}$ W. T. Ford, ${ }^{20}$ J. F. Hirschauer, ${ }^{20}$ A. Kreisel, ${ }^{20}$ M. Nagel, ${ }^{20}$ U. Nauenberg, ${ }^{20}$ A. Olivas, ${ }^{20}$ J. G. Smith,${ }^{20}$ K. A. Ulmer, ${ }^{20}$ S. R. Wagner, ${ }^{20}$ J. Zhang, ${ }^{20}$ A. M. Gabareen, ${ }^{21}$ A. Soffer ${ }^{21}$ W. H. Toki, ${ }^{21}$ R. J. Wilson, ${ }^{21}$ F. Winklmeier, ${ }^{21}$ Q. Zeng, ${ }^{21}$ D. D. Altenburg, ${ }^{22}$ E. Feltresi, ${ }^{22}$ A. Hauke, ${ }^{22}$ H. Jasper, ${ }^{22}$ J. Merkel, ${ }^{22}$ A. Petzold, ${ }^{22}$ B. Spaan, ${ }^{22}$ K. Wacker, ${ }^{22}$ T. Brandt,${ }^{23}$ V. Klose, ${ }^{23}$ M. J. Kobel, ${ }^{23}$ H. M. Lacker, ${ }^{23}$ W. F. Mader, ${ }^{23}$ R. Nogowski, ${ }^{23}$ J. Schubert, ${ }^{23}$ K. R. Schubert, ${ }^{23}$ R. Schwierz, ${ }^{23}$

J. E. Sundermann, ${ }^{23}$ A. Volk, ${ }^{23}$ D. Bernard, ${ }^{24}$ G. R. Bonneaud, ${ }^{24}$ E. Latour, ${ }^{24}$ V. Lombardo, ${ }^{24}$ Ch. Thiebaux, ${ }^{24}$ M. Verderi, ${ }^{24}$ P. J. Clark,${ }^{25}$ W. Gradl,${ }^{25}$ F. Muheim, ${ }^{25}$ S. Playfer, ${ }^{25}$ A. I. Robertson, ${ }^{25}$ Y. Xie, ${ }^{25}$ M. Andreotti, ${ }^{26}$ D. Bettoni, ${ }^{26}$ C. Bozzi,${ }^{26}$ R. Calabrese, ${ }^{26}$ A. Cecchi, ${ }^{26}$ G. Cibinetto, ${ }^{26}$ P. Franchini, ${ }^{26}$ E. Luppi ${ }^{26}$ M. Negrini, ${ }^{26}$ A. Petrella, ${ }^{26}$ L. Piemontese, ${ }^{26}$ E. Prencipe, ${ }^{26}$ V. Santoro,${ }^{26}$ F. Anulli, ${ }^{27}$ R. Baldini-Ferroli, ${ }^{27}$ A. Calcaterra, ${ }^{27}$ R. de Sangro, ${ }^{27}$ G. Finocchiaro, ${ }^{27}$ S. Pacetti, ${ }^{27}$ P. Patteri, ${ }^{27}$ I. M. Peruzzi, ${ }^{27, \dagger}$ M. Piccolo,${ }^{27}$ M. Rama, ${ }^{27}$ A. Zallo, ${ }^{27}$ A. Buzzo, ${ }^{28}$ R. Contri, ${ }^{28}$ M. Lo Vetere, ${ }^{28}$ M. M. Macri, ${ }^{28}$ M. R. Monge, ${ }^{28}$ S. Passaggio, ${ }^{28}$ C. Patrignani,${ }^{28}$ E. Robutti, ${ }^{28}$ A. Santroni, ${ }^{28}$ S. Tosi, ${ }^{28}$ K. S. Chaisanguanthum, ${ }^{29}$ M. Morii, ${ }^{29}$ J. Wu ${ }^{29}$ R. S. Dubitzky, ${ }^{30}$ J. Marks,${ }^{30}$ S. Schenk, ${ }^{30}$ U. Uwer, ${ }^{30}$ D. J. Bard, ${ }^{31}$ P. D. Dauncey, ${ }^{31}$ R. L. Flack, ${ }^{31}$ J. A. Nash, ${ }^{31}$ M. B. Nikolich, ${ }^{31}$ W. Panduro Vazquez, ${ }^{31}$ P. K. Behera, ${ }^{32}$ X. Chai, ${ }^{32}$ M. J. Charles ${ }^{32}$ U. Mallik, ${ }^{32}$ N. T. Meyer, ${ }^{32}$ V. Ziegler, ${ }^{32}$ J. Cochran, ${ }^{33}$ H. B. Crawley, ${ }^{33}$ L. Dong, ${ }^{33}$ V. Eyges, ${ }^{33}$ W. T. Meyer, ${ }^{33}$ S. Prell, ${ }^{33}$ E. I. Rosenberg, ${ }^{33}$ A. E. Rubin, ${ }^{33}$ A. V. Gritsan, ${ }^{34}$ Z. J. Guo, ${ }^{34}$ C. K. Lae, ${ }^{34}$ A. G. Denig, ${ }^{35}$ M. Fritsch, ${ }^{35}$ G. Schott, ${ }^{35}$ N. Arnaud, ${ }^{36}$ J. Béquilleux, ${ }^{36}$ M. Davier, ${ }^{36}$ G. Grosdidier, ${ }^{36}$ A. Höcker, ${ }^{36}$ V. Lepeltier, ${ }^{36}$ F. Le Diberder, ${ }^{36}$ A. M. Lutz, ${ }^{36}$ S. Pruvot, ${ }^{36}$ S. Rodier, ${ }^{36}$ P. Roudeau, ${ }^{36}$ M. H. Schune, ${ }^{36}$ J. Serrano, ${ }^{36}$ V. Sordini, ${ }^{36}$ A. Stocchi, ${ }^{36}$ W. F. Wang, ${ }^{36}$ G. Wormser, ${ }^{36}$ D. J. Lange, ${ }^{37}$ D. M. Wright, ${ }^{37}$ C. A. Chavez, ${ }^{38}$ I. J. Forster, ${ }^{38}$ J. R. Fry ${ }^{38}$ E. Gabathuler, ${ }^{38}$ R. Gamet, ${ }^{38}$ D. E. Hutchcroft, ${ }^{38}$ D. J. Payne, ${ }^{38}$ K. C. Schofield ${ }^{38}$ C. Touramanis, ${ }^{38}$ A. J. Bevan, ${ }^{39}$ K. A. George, ${ }^{39}$ F. Di Lodovico, ${ }^{39}$ W. Menges, ${ }^{39}$ R. Sacco, ${ }^{39}$ G. Cowan, ${ }^{40}$ H. U. Flaecher, ${ }^{40}$ D. A. Hopkins, ${ }^{40}$ P. S. Jackson, ${ }^{40}$ T. R. McMahon, ${ }^{40}$ F. Salvatore, ${ }^{40}$ A. C. Wren, ${ }^{40}$ D. N. Brown, ${ }^{41}$ C. L. Davis, ${ }^{41}$ J. Allison,${ }^{42}$ N. R. Barlow, ${ }^{42}$ R. J. Barlow, ${ }^{42}$ Y. M. Chia, ${ }^{42}$ C. L. Edgar, ${ }^{42}$ G. D. Lafferty, ${ }^{42}$ T. J. West, ${ }^{42}$ J. I. Yi, ${ }^{42}$ J. Anderson, ${ }^{43}$ C. Chen, ${ }^{43}$

A. Jawahery, ${ }^{43}$ D. A. Roberts, ${ }^{43}$ G. Simi, ${ }^{43}$ J. M. Tuggle, ${ }^{43}$ G. Blaylock, ${ }^{44}$ C. Dallapiccola, ${ }^{44}$ S. S. Hertzbach, ${ }^{44}$ X. Li ${ }^{44}$

T. B. Moore, ${ }^{44}$ E. Salvati, ${ }^{44}$ S. Saremi, ${ }^{44}$ R. Cowan,${ }^{45}$ P. H. Fisher,${ }^{45}$ G. Sciolla,${ }^{45}$ S. J. Sekula, ${ }^{45}$ M. Spitznagel ${ }^{45}$

F. Taylor, ${ }^{45}$ R. K. Yamamoto, ${ }^{45}$ S. E. Mclachlin ${ }^{46}$ P. M. Patel, ${ }^{46}$ S. H. Robertson, ${ }^{46}$ A. Lazzaro, ${ }^{47}$ F. Palombo, ${ }^{47}$

J. M. Bauer, ${ }^{48}$ L. Cremaldi, ${ }^{48}$ V. Eschenburg, ${ }^{48}$ R. Godang, ${ }^{48}$ R. Kroeger, ${ }^{48}$ D. A. Sanders, ${ }^{48}$ D. J. Summers, ${ }^{48}$ H. W. Zhao, ${ }^{48}$ S. Brunet, ${ }^{49}$ D. Côté,${ }^{49}$ M. Simard, ${ }^{49}$ P. Taras,${ }^{49}$ F. B. Viaud, ${ }^{49}$ H. Nicholson, ${ }^{50}$ G. De Nardo,${ }^{51}$ F. Fabozzi, ${ }^{51,}$ L. Lista, ${ }^{51}$ D. Monorchio, ${ }^{51}$ C. Sciacca,${ }^{51}$ M. A. Baak, ${ }^{52}$ G. Raven, ${ }^{52}$ H. L. Snoek, ${ }^{52}$ C. P. Jessop, ${ }^{53}$ J. M. LoSecco, ${ }^{53}$ G. Benelli, ${ }^{54}$ L. A. Corwin, ${ }^{54}$ K. K. Gan, ${ }^{54}$ K. Honscheid,${ }^{54}$ D. Hufnagel,${ }^{54}$ H. Kagan, ${ }^{54}$ R. Kass,${ }^{54}$ J. P. Morris, ${ }^{54}$ A. M. Rahimi, ${ }^{54}$ J. J. Regensburger, ${ }^{54}$ R. Ter-Antonyan,${ }^{54}$ Q. K. Wong, ${ }^{54}$ N. L. Blount, ${ }^{55}$ J. Brau, ${ }^{55}$ R. Frey,${ }^{55}$ O. Igonkina, ${ }^{55}$ J. A. Kolb, ${ }^{55}$ M. Lu, ${ }^{55}$ R. Rahmat, ${ }^{55}$ N. B. Sinev, ${ }^{55}$ D. Strom, ${ }^{55}$ J. Strube, ${ }^{55}$ E. Torrence, ${ }^{55}$ N. Gagliardi, ${ }^{56}$ A. Gaz, ${ }^{56}$ M. Margoni, ${ }^{56}$ M. Morandin, ${ }^{56}$ A. Pompili, ${ }^{56}$ M. Posocco,${ }^{56}$ M. Rotondo,${ }^{56}$ F. Simonetto, ${ }^{56}$ R. Stroili, ${ }^{56}$ C. Voci, ${ }^{56}$ E. Ben-Haim,${ }^{57}$ H. Briand ${ }^{57}$ G. Calderini, ${ }^{57}$ J. Chauveau, ${ }^{57}$ P. David,${ }^{57}$ L. Del Buono, ${ }^{57}$ Ch. de la Vaissière, ${ }^{57}$ O. Hamon,${ }^{57}$ Ph. Leruste, ${ }^{57}$ J. Malclès, ${ }^{57}$ J. Ocariz,${ }^{57}$ A. Perez,${ }^{57}$ L. Gladney,${ }^{58}$ M. Biasini, ${ }^{59}$ R. Covarelli, ${ }^{59}$ E. Manoni,${ }^{59}$ C. Angelini, ${ }^{60}$ G. Batignani, ${ }^{60}$ S. Bettarini, ${ }^{60}$ M. Carpinelli, ${ }^{60}$ R. Cenci, ${ }^{60}$ A. Cervelli, ${ }^{60}$ F. Forti, ${ }^{60}$ M. A. Giorgi, ${ }^{60}$ A. Lusiani, ${ }^{60}$ 
G. Marchiori, ${ }^{60}$ M. A. Mazur, ${ }^{60}$ M. Morganti,${ }^{60}$ N. Neri, ${ }^{60}$ E. Paoloni, ${ }^{60}$ G. Rizzo,${ }^{60}$ J. J. Walsh,${ }^{60}$ M. Haire,${ }^{61}$ J. Biesiada, ${ }^{62}$ P. Elmer, ${ }^{62}$ Y. P. Lau, ${ }^{62}$ C. Lu, ${ }^{62}$ J. Olsen, ${ }^{62}$ A. J. S. Smith, ${ }^{62}$ A. V. Telnov, ${ }^{62}$ E. Baracchini, ${ }^{63}$ F. Bellini, ${ }^{63}$ G. Cavoto, ${ }^{63}$ A. D’Orazio, ${ }^{63}$ D. del Re ${ }^{63}$ E. Di Marco,${ }^{63}$ R. Faccini, ${ }^{63}$ F. Ferrarotto, ${ }^{63}$ F. Ferroni, ${ }^{63}$ M. Gaspero, ${ }^{63}$ P. D. Jackson, ${ }^{63}$ L. Li Gioi, ${ }^{63}$ M. A. Mazzoni, ${ }^{63}$ S. Morganti, ${ }^{63}$ G. Piredda, ${ }^{63}$ F. Polci, ${ }^{63}$ F. Renga ${ }^{63}$ C. Voena,${ }^{63}$ M. Ebert, ${ }^{64}$ H. Schröder,${ }^{64}$ R. Waldi, ${ }^{64}$ T. Adye, ${ }^{65}$ G. Castelli, ${ }^{65}$ B. Franek, ${ }^{65}$ E. O. Olaiya, ${ }^{65}$ S. Ricciardi ${ }^{65}$ W. Roethel,${ }^{65}$ F. F. Wilson, ${ }^{65}$ R. Aleksan, ${ }^{66}$ S. Emery, ${ }^{66}$ M. Escalier, ${ }^{66}$ A. Gaidot, ${ }^{66}$ S. F. Ganzhur, ${ }^{66}$ G. Hamel de Monchenault, ${ }^{66}$ W. Kozanecki, ${ }^{66}$ M. Legendre, ${ }^{66}$ G. Vasseur, ${ }^{66}$ Ch. Yèche, ${ }^{66}$ M. Zito, ${ }^{66}$ X. R. Chen, ${ }^{67}$ H. Liu, ${ }^{67}$ W. Park, ${ }^{67}$ M. V. Purohit, ${ }^{67}$ J. R. Wilson, ${ }^{67}$ M. T. Allen, ${ }^{68}$ D. Aston, ${ }^{68}$ R. Bartoldus, ${ }^{68}$ P. Bechtle, ${ }^{68}$ N. Berger, ${ }^{68}$ R. Claus, ${ }^{68}$ J. P. Coleman ${ }^{68}$ M. R. Convery, ${ }^{68}$ J. C. Dingfelder, ${ }^{68}$ J. Dorfan, ${ }^{68}$ G. P. Dubois-Felsmann, ${ }^{68}$ D. Dujmic, ${ }^{68}$ W. Dunwoodie ${ }^{68}$ R. C. Field, ${ }^{68}$ T. Glanzman, ${ }^{68}$ S. J. Gowdy, ${ }^{68}$ M. T. Graham, ${ }^{68}$ P. Grenier, ${ }^{68}$ C. Hast, ${ }^{68}$ T. Hryn'ova, ${ }^{68}$ W. R. Innes, ${ }^{68}$ J. Kaminski, ${ }^{68}$ M. H. Kelsey, ${ }^{68}$ H. Kim, ${ }^{68}$ P. Kim, ${ }^{68}$ M. L. Kocian, ${ }^{68}$ D. W. G. S. Leith, ${ }^{68}$ S. Li,${ }^{68}$ S. Luitz, ${ }^{68}$ V. Luth ${ }^{68}$ H. L. Lynch, ${ }^{68}$ D. B. MacFarlane,${ }^{68}$ H. Marsiske, ${ }^{68}$ R. Messner, ${ }^{68}$ D. R. Muller, ${ }^{68}$ C. P. O' Grady ${ }^{68}{ }^{6}$. Ofte, ${ }^{68}$ A. Perazzo, ${ }^{68}$ M. Perl, ${ }^{68}$ T. Pulliam, ${ }^{68}$ B. N. Ratcliff, ${ }^{68}$ A. Roodman, ${ }^{68}$ A. A. Salnikov, ${ }^{68}$ R. H. Schindler, ${ }^{68}$ J. Schwiening, ${ }^{68}$ A. Snyder, ${ }^{68}$ J. Stelzer, ${ }^{68}$ D. Su, ${ }^{68}$ M. K. Sullivan, ${ }^{68}$ K. Suzuki, ${ }^{68}$ S. K. Swain, ${ }^{68}$ J. M. Thompson, ${ }^{68}$ J. Va'vra, ${ }^{68}$ N. van Bakel, ${ }^{68}$ A. P. Wagner, ${ }^{68}$ M. Weaver, ${ }^{68}$ W. J. Wisniewski, ${ }^{68}$ M. Wittgen, ${ }^{68}$ D. H. Wright, ${ }^{68}$ A. K. Yarritu, ${ }^{68}$ K. Yi, ${ }^{68}$ C. C. Young, ${ }^{68}$ P. R. Burchat, ${ }^{69}$ A. J. Edwards,${ }^{69}$ S. A. Majewski, ${ }^{69}$ B. A. Petersen, ${ }^{69}$ L. Wilden, ${ }^{69}$ S. Ahmed ${ }^{70}$ M. S. Alam,${ }^{70}$ R. Bula, ${ }^{70}$ J. A. Ernst,${ }^{70}$ V. Jain, ${ }^{70}$ B. Pan, ${ }^{70}$ M. A. Saeed, ${ }^{70}$ F. R. Wappler, ${ }^{70}$ S. B. Zain, ${ }^{70}$ W. Bugg, ${ }^{71}$ M. Krishnamurthy, ${ }^{71}$ S. M. Spanier, ${ }^{71}$ R. Eckmann, ${ }^{72}$ J. L. Ritchie, ${ }^{72}$ A. M. Ruland, ${ }^{72}$ C. J. Schilling, ${ }^{72}$ R. F. Schwitters, ${ }^{72}$ J. M. Izen, ${ }^{73}$ X. C. Lou, ${ }^{73}$ S. Ye, ${ }^{73}$ F. Bianchi, ${ }^{74}$ F. Gallo, ${ }^{74}$ D. Gamba, ${ }^{74}$ M. Pelliccioni,${ }^{74}$ M. Bomben, ${ }^{75}$ L. Bosisio,${ }^{75}$ C. Cartaro, ${ }^{75}$ F. Cossutti, ${ }^{75}$ G. Della Ricca, ${ }^{75}$ L. Lanceri, ${ }^{75}$ L. Vitale, ${ }^{75}$ V. Azzolini, ${ }^{76}$ N. Lopez-March, ${ }^{76}$ F. Martinez-Vidal, ${ }^{76}$ D. A. Milanes, ${ }^{76}$ A. Oyanguren, ${ }^{76}$ J. Albert, ${ }^{77}$ Sw. Banerjee, ${ }^{77}$ B. Bhuyan, ${ }^{77}$ K. Hamano,${ }^{77}$ R. Kowalewski, ${ }^{77}$ I. M. Nugent, ${ }^{77}$ J. M. Roney, ${ }^{77}$ R. J. Sobie, ${ }^{77}$ J. J. Back, ${ }^{78}$ P. F. Harrison, ${ }^{78}$ T. E. Latham, ${ }^{78}$ G. B. Mohanty, ${ }^{78}$ M. Pappagallo, ${ }^{78,8}$ H. R. Band ${ }^{79}$ X. Chen, ${ }^{79}$ S. Dasu ${ }^{79}$ K. T. Flood, ${ }^{79}$ J. J. Hollar, ${ }^{79}$ P. E. Kutter, ${ }^{79}$ Y. Pan, ${ }^{79}$ M. Pierini, ${ }^{79}$ R. Prepost, ${ }^{79}$ S. L. Wu, ${ }^{79}$ Z. Yu, ${ }^{79}$ and H. Neal ${ }^{80}$

(BABAR Collaboration)

\footnotetext{
${ }^{1}$ Laboratoire de Physique des Particules, IN2P3/CNRS et Université de Savoie, F-74941 Annecy-Le-Vieux, France

${ }^{2}$ Universitat de Barcelona, Facultat de Fisica, Departament ECM, E-08028 Barcelona, Spain

${ }^{3}$ Università di Bari, Dipartimento di Fisica and INFN, I-70126 Bari, Italy

${ }^{4}$ University of Bergen, Institute of Physics, N-5007 Bergen, Norway

${ }^{5}$ Lawrence Berkeley National Laboratory and University of California, Berkeley, California 94720, USA

${ }^{6}$ University of Birmingham, Birmingham, B15 2TT, United Kingdom

${ }^{7}$ Ruhr Universität Bochum, Institut für Experimentalphysik 1, D-44780 Bochum, Germany

${ }^{8}$ University of Bristol, Bristol BS8 1TL, United Kingdom

${ }^{9}$ University of British Columbia, Vancouver, British Columbia, Canada V6T 1 Z1

${ }^{10}$ Brunel University, Uxbridge, Middlesex UB8 3PH, United Kingdom

${ }^{11}$ Budker Institute of Nuclear Physics, Novosibirsk 630090, Russia

${ }^{12}$ University of California at Irvine, Irvine, California 92697, USA

${ }^{13}$ University of California at Los Angeles, Los Angeles, California 90024, USA

${ }^{14}$ University of California at Riverside, Riverside, California 92521, USA

${ }^{15}$ University of California at San Diego, La Jolla, California 92093, USA

${ }^{16}$ University of California at Santa Barbara, Santa Barbara, California 93106, USA

${ }^{17}$ University of California at Santa Cruz, Institute for Particle Physics, Santa Cruz, California 95064, USA

${ }^{18}$ California Institute of Technology, Pasadena, California 91125, USA

${ }^{19}$ University of Cincinnati, Cincinnati, Ohio 45221, USA

${ }^{20}$ University of Colorado, Boulder, Colorado 80309, USA

${ }^{21}$ Colorado State University, Fort Collins, Colorado 80523, USA

${ }^{22}$ Universität Dortmund, Institut für Physik, D-44221 Dortmund, Germany

${ }^{23}$ Technische Universität Dresden, Institut für Kern-und Teilchenphysik, D-01062 Dresden, Germany

${ }^{24}$ Laboratoire Leprince-Ringuet, CNRS/IN2P3, Ecole Polytechnique, F-91128 Palaiseau, France

${ }^{25}$ University of Edinburgh, Edinburgh EH9 3JZ, United Kingdom

${ }^{26}$ Università di Ferrara, Dipartimento di Fisica and INFN, I-44100 Ferrara, Italy

${ }^{27}$ Laboratori Nazionali di Frascati dell'INFN, I-00044 Frascati, Italy

${ }^{28}$ Università di Genova, Dipartimento di Fisica and INFN, I-16146 Genova, Italy

${ }^{29}$ Harvard University, Cambridge, Massachusetts 02138, USA

${ }^{30}$ Universität Heidelberg, Physikalisches Institut, Philosophenweg 12, D-69120 Heidelberg, Germany
} 
${ }^{31}$ Imperial College London, London, SW7 2AZ, United Kingdom

${ }^{32}$ University of Iowa, Iowa City, Iowa 52242, USA

${ }^{33}$ Iowa State University, Ames, Iowa 50011-3160, USA

${ }^{34}$ Johns Hopkins University, Baltimore, Maryland 21218, USA

${ }^{35}$ Universität Karlsruhe, Institut für Experimentelle Kernphysik, D-76021 Karlsruhe, Germany

${ }^{36}$ Laboratoire de l'Accélérateur Linéaire, IN2P3/CNRS et Université Paris-Sud 11, Centre Scientifique d'Orsay, B.P. 34, F-91898 ORSAY Cedex, France

${ }^{37}$ Lawrence Livermore National Laboratory, Livermore, California 94550, USA

${ }^{38}$ University of Liverpool, Liverpool L69 7ZE, United Kingdom

${ }^{39}$ Queen Mary, University of London, E1 4NS, United Kingdom

${ }^{40}$ University of London, Royal Holloway and Bedford New College, Egham, Surrey TW20 OEX, United Kingdom

${ }^{41}$ University of Louisville, Louisville, Kentucky 40292, USA

${ }^{42}$ University of Manchester, Manchester M13 9PL, United Kingdom

${ }^{43}$ University of Maryland, College Park, Maryland 20742, USA

${ }^{44}$ University of Massachusetts, Amherst, Massachusetts 01003, USA

${ }^{45}$ Massachusetts Institute of Technology, Laboratory for Nuclear Science, Cambridge, Massachusetts 02139, USA

${ }^{46}$ McGill University, Montréal, Québec, Canada H3A $2 T 8$

${ }^{47}$ Università di Milano, Dipartimento di Fisica and INFN, I-20133 Milano, Italy

${ }^{48}$ University of Mississippi, University, Mississippi 38677, USA

${ }^{49}$ Université de Montréal, Physique des Particules, Montréal, Québec, Canada H3C 3J7

${ }^{50}$ Mount Holyoke College, South Hadley, Massachusetts 01075, USA

${ }^{51}$ Università di Napoli Federico II, Dipartimento di Scienze Fisiche and INFN, I-80126, Napoli, Italy

${ }^{52}$ NIKHEF, National Institute for Nuclear Physics and High Energy Physics, NL-1009 DB Amsterdam, The Netherlands

${ }^{53}$ University of Notre Dame, Notre Dame, Indiana 46556, USA

${ }^{54}$ Ohio State University, Columbus, Ohio 43210, USA

${ }^{55}$ University of Oregon, Eugene, Oregon 97403, USA

${ }^{56}$ Università di Padova, Dipartimento di Fisica and INFN, I-35131 Padova, Italy

${ }^{57}$ Laboratoire de Physique Nucléaire et de Hautes Energies, IN2P3/CNRS, Université Pierre et Marie Curie-Paris6, Université Denis Diderot-Paris7, F-75252 Paris, France

${ }^{58}$ University of Pennsylvania, Philadelphia, Pennsylvania 19104, USA

${ }^{59}$ Università di Perugia, Dipartimento di Fisica and INFN, I-06100 Perugia, Italy

${ }^{60}$ Università di Pisa, Dipartimento di Fisica, Scuola Normale Superiore and INFN, I-56127 Pisa, Italy

${ }^{61}$ Prairie View A\&M University, Prairie View, Texas 77446, USA

${ }^{62}$ Princeton University, Princeton, New Jersey 08544, USA

${ }^{63}$ Università di Roma La Sapienza, Dipartimento di Fisica and INFN, I-00185 Roma, Italy

${ }^{64}$ Universität Rostock, D-18051 Rostock, Germany

${ }^{65}$ Rutherford Appleton Laboratory, Chilton, Didcot, Oxon, OX11 0QX, United Kingdom

${ }^{66}$ DSM/Dapnia, CEA/Saclay, F-91191 Gif-sur-Yvette, France

${ }^{67}$ University of South Carolina, Columbia, South Carolina 29208, USA

${ }^{68}$ Stanford Linear Accelerator Center, Stanford, California 94309, USA

${ }^{69}$ Stanford University, Stanford, California 94305-4060, USA

${ }^{70}$ State University of New York, Albany, New York 12222, USA

${ }^{71}$ University of Tennessee, Knoxville, Tennessee 37996, USA

${ }^{72}$ University of Texas at Austin, Austin, Texas 78712, USA

${ }^{73}$ University of Texas at Dallas, Richardson, Texas 75083, USA

${ }^{74}$ Università di Torino, Dipartimento di Fisica Sperimentale and INFN, I-10125 Torino, Italy

${ }^{75}$ Università di Trieste, Dipartimento di Fisica and INFN, I-34127 Trieste, Italy

${ }^{76}$ IFIC, Universitat de Valencia-CSIC, E-46071 Valencia, Spain

${ }^{77}$ University of Victoria, Victoria, British Columbia, Canada V8W $3 P 6$

${ }^{78}$ Department of Physics, University of Warwick, Coventry CV4 7AL, United Kingdom

${ }^{79}$ University of Wisconsin, Madison, Wisconsin 53706, USA

${ }^{80}$ Yale University, New Haven, Connecticut 06511, USA

(Received 26 April 2007; published 9 July 2007)

Using $385 \mathrm{fb}^{-1}$ of $e^{+} e^{-}$collisions, we study the amplitudes of the singly Cabibbo-suppressed decay $D^{0} \rightarrow K^{-} K^{+} \pi^{0}$. We measure the strong phase difference between the $\bar{D}^{0}$ and $D^{0}$ decays to $K^{*}(892)^{+} K^{-}$

\footnotetext{
*Deceased

†Also with Università di Perugia, Dipartimento di Fisica, Perugia, Italy.

*Also with Università della Basilicata, Potenza, Italy.

${ }^{\S}$ Also with IPPP, Physics Department, Durham University, Durham DH1 3LE, United Kingdom.
} 
to be $-35.5^{\circ} \pm 1.9^{\circ}$ (stat) $\pm 2.2^{\circ}$ (syst), and their amplitude ratio to be $0.599 \pm 0.013$ (stat) \pm 0.011 (syst). We observe contributions from the $K \pi$ and $K^{-} K^{+}$scalar and vector amplitudes, and analyze their angular moments. We find no evidence for charged $\kappa$, nor for higher spin states. We also perform a partial-wave analysis of the $K^{-} K^{+}$system in a limited mass range.

The amplitudes describing $D$ meson weak decays into three-body final states are dominated by intermediate resonances that lead to highly nonuniform intensity distributions in the available phase space. Analyses of these distributions have led to new insights into the role of the light-meson systems produced [1]. The $K^{ \pm} \pi^{0}$ systems from the decay $D^{0} \rightarrow K^{-} K^{+} \pi^{0}$ [2] can provide information on the $K \pi S$-wave (spin-0) amplitude in the mass range $0.6-1.4 \mathrm{GeV} / c^{2}$, and hence on the possible existence of the $\kappa(800)$, reported to date only in the neutral state $\left(\kappa^{0} \rightarrow K^{-} \pi^{+}\right)$[3]. If the $\kappa$ has isospin $1 / 2$, it should be observable also in the charged states. Results of the present analysis can be an input for extracting the $C P$-violating phase $\gamma=\arg \left(-V_{u d} V_{u b}^{*} / V_{c d} V_{c b}^{*}\right)$ of the quark mixing matrix by exploiting interference structure in the Dalitz plot from the decay $B^{ \pm} \rightarrow D_{K^{-} K^{+} \pi^{0}}^{0} K^{ \pm}[4,5]$. Singly Cabibbo-suppressed decays are also important because they might be sensitive to direct $C P$ violation in charm decays [6], the discovery of which might indicate physics beyond the standard model.

We perform the present analysis on $385 \mathrm{fb}^{-1}$ of $e^{+} e^{-}$ collision data collected at and around $10.58 \mathrm{GeV}$ center-ofmass (CM) energy with the BABAR detector [7] at the PEPII storage ring. We distinguish $D^{0}$ from $\bar{D}^{0}$ by reconstructing the decays $D^{*+} \rightarrow D^{0} \pi^{+}$and $D^{*-} \rightarrow \bar{D}^{0} \pi^{-}$. The event-selection criteria are the same as those used in our measurement of the branching ratio of the decay $D^{0} \rightarrow$ $K^{-} K^{+} \pi^{0}$ [8]. In particular, we require that the CM momentum of the $D^{0}$ candidate be greater than $2.77 \mathrm{GeV} / c$, and that $\left|m_{D^{*+}}-m_{D^{0}}-145.4\right|<0.6 \mathrm{MeV} / c^{2}$, where $m$ refers to a reconstructed invariant mass. To minimize uncertainty from background shape, we choose a sample of very high purity $(\sim 98.1 \%)$ using $1855<m_{D^{0}}<$ $1875 \mathrm{MeV} / c^{2}$, and find $11278 \pm 110$ signal events. We estimate the signal efficiency for each event as a function of its position in the Dalitz plot using simulated $D^{0} \rightarrow$ $K^{-} K^{+} \pi^{0}$ events from $c \bar{c}$ decays, generated uniformly in the available phase space. To correct for differences in particle-identification rates in data and simulation, we determine the ratio of these for each track and apply an event-by-event correction factor.

Neglecting $C P$ violation in $D$ meson decays, we define the $D^{0}\left(\bar{D}^{0}\right)$ decay amplitude $\mathcal{A}(\overline{\mathcal{A}})$ in the $D^{0} \rightarrow$ $K^{-} K^{+} \pi^{0}$ Dalitz plot of Fig. 1, as

$$
\begin{aligned}
& \mathcal{A}\left[D^{0} \rightarrow K^{-} K^{+} \pi^{0}\right] \equiv f_{D^{0}}\left(m_{K^{+} \pi^{0}}^{2}, m_{K^{-} \pi^{0}}^{2}\right), \\
& \overline{\mathcal{A}}\left[\bar{D}^{0} \rightarrow K^{+} K^{-} \pi^{0}\right] \equiv f_{D^{0}}\left(m_{K^{-} \pi^{0}}^{2}, m_{K^{+} \pi^{0}}^{2}\right) .
\end{aligned}
$$

The complex quantum mechanical amplitude $f$ is a coherent sum of all relevant quasi-two-body $D^{0} \rightarrow(r \rightarrow A B) C$ isobar model [9] resonances, $f=\sum_{r} a_{r} e^{i \phi_{r}} A_{r}(s)$. Here $s=$ $m_{A B}^{2}$, and $A_{r}$ is the resonance amplitude. We obtain coefficients $a_{r}$ and $\phi_{r}$ from a likelihood fit. The probability density function for signal events is $|f|^{2}$. We model incoherent background empirically using events from the lower sideband of the $m_{D^{0}}$ [8] distribution.

For $D^{0}$ decays to spin-1 ( $P$-wave) and spin-2 states, we use the Breit-Wigner amplitude,

$$
\begin{gathered}
A_{\mathrm{BW}}(s)=\mathcal{M}_{L}(s, p) \frac{1}{M_{0}^{2}-s-i M_{0} \Gamma(s)}, \\
\Gamma(s)=\Gamma_{0}\left(\frac{M_{0}}{\sqrt{s}}\right)\left(\frac{p}{p_{0}}\right)^{2 L+1}\left[\frac{\mathcal{F}_{L}(p)}{\mathcal{F}_{L}\left(p_{0}\right)}\right]^{2},
\end{gathered}
$$

where $M_{0}\left(\Gamma_{0}\right)$ is the resonance mass (width) [10], $L$ is the angular momentum quantum number, $p$ is the momentum of either daughter in the resonance rest frame, and $p_{0}$ is the value of $p$ when $s=M_{0}^{2}$. The function $\mathcal{F}_{L}$ is the Blatt-
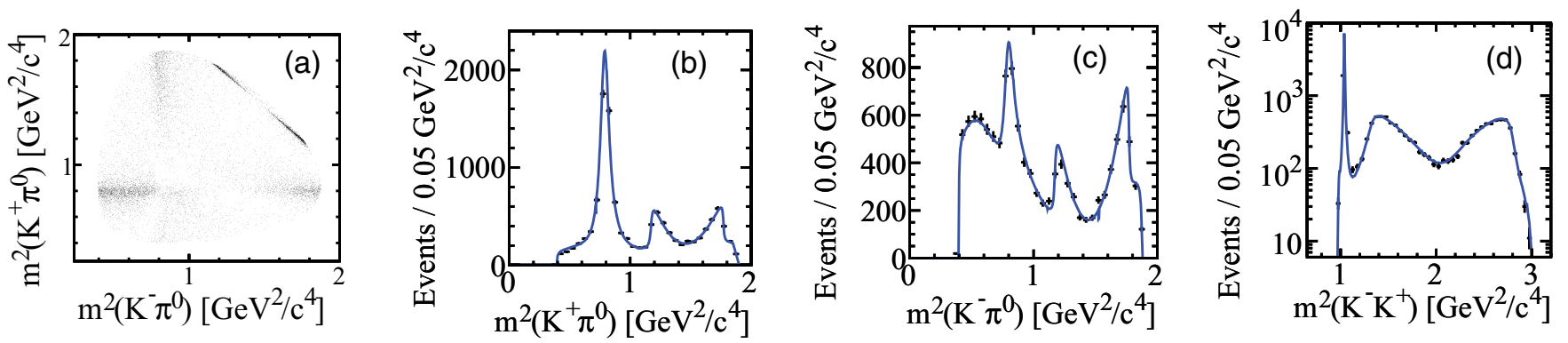

FIG. 1 (color online). Dalitz plot for $D^{0} \rightarrow K^{-} K^{+} \pi^{0}$ [2] data (a), and the corresponding squared invariant mass projections (b)-(d). The three-body invariant mass of the $D^{0}$ candidate is constrained to the nominal value. In plots (b) - (d), the dots (with error bars, black) are data points and the solid lines (blue) correspond to the best isobar fit models. 
Weisskopf barrier factor [11]: $\mathcal{F}_{0}=1, \mathcal{F}_{1}=1 / \sqrt{1+R p^{2}}$, and $\mathcal{F}_{2}=1 / \sqrt{9+3 R p^{2}+R p^{4}}$, where we take the meson radial parameter $R$ to be $1.5 \mathrm{GeV}^{-1}$ [12]. We define the spin part of the amplitude, $\mathcal{M}_{L}$, as $\mathcal{M}_{0}=M_{D^{0}}^{2}, \mathcal{M}_{1}=$ $-2 \vec{p}_{A} \cdot \vec{p}_{C}$, and $\mathcal{M}_{2}=\frac{4}{3}\left[3\left(\vec{p}_{A} \cdot \vec{p}_{C}\right)^{2}-\left|\vec{p}_{A}\right|^{2} \cdot\left|\vec{p}_{C}\right|^{2}\right] M_{D^{0}}^{-2}$, where $M_{D^{0}}$ is the nominal $D^{0}$ mass, and $\vec{p}_{i}$ is the 3momentum of particle $i$ in the resonance rest frame.

For $D^{0}$ decays to $K^{ \pm} \pi^{0} S$-wave states, we consider three amplitude models. One model uses the LASS amplitude for $K^{-} \pi^{+} \rightarrow K^{-} \pi^{+}$elastic scattering [13],

$$
\begin{gathered}
A_{K \pi(S)}(s)=\frac{\sqrt{s}}{p} \sin \delta(s) e^{i \delta(s),} \\
\delta(s)=\cot ^{-1}\left(\frac{1}{p a}+\frac{b p}{2}\right)+\cot ^{-1}\left(\frac{M_{0}^{2}-s}{M_{0} \Gamma_{0} \cdot \frac{M_{0}}{\sqrt{s}} \cdot \frac{p}{p_{0}}}\right),
\end{gathered}
$$

where $M_{0}\left(\Gamma_{0}\right)$ refers to the $K_{0}^{*}(1430)$ mass (width), $a=$ $1.95 \pm 0.09 \mathrm{GeV}^{-1} c$, and $b=1.76 \pm 0.36 \mathrm{GeV}^{-1} c$. The unitary nature of Eq. (5) provides a good description of the amplitude up to $1.45 \mathrm{GeV} / c^{2}$ (i.e., $K \eta^{\prime}$ threshold). In Eq. (6), the first term is a nonresonant contribution defined by a scattering length $a$ and an effective range $b$, and the second term represents the $K_{0}^{*}(1430)$ resonance. The phase space factor $\sqrt{s} / p$ converts the scattering amplitude to the invariant amplitude. Our second model uses the E-791 results for the $K^{-} \pi^{+} S$-wave amplitude from an energyindependent partial-wave analysis in the decay $D^{+} \rightarrow$ $K^{-} \pi^{+} \pi^{+}$[14]. The third model uses a coherent sum of a uniform nonresonant term, and Breit-Wigner terms for the $\kappa(800)$ and $K_{0}^{*}(1430)$ resonances.

In Fig. 2 we compare the $K \pi S$-wave amplitude from the E-791 analysis [14] to the LASS amplitude of Eqs. (5) and (6). For easy comparison, we have normalized the LASS amplitude in Fig. 2(a) approximately to the E-791 measurements with $\sqrt{s}>1.15 \mathrm{GeV} / c^{2}$, and have reduced the LASS phase, $\delta(s)$, in Fig. 2(b) by $80^{\circ}$. We then observe good agreements in the mass dependence of amplitude and phase for $\sqrt{s}>1.15 \mathrm{GeV} / c^{2}$. As the mass decreases from $1.15 \mathrm{GeV} / c^{2}$, the E-791 amplitude increases while the LASS amplitude decreases, with the ratio finally reaching $\sim 1.7$ at threshold. At the same time, their phase difference increases to $\sim 40^{\circ}$ at threshold. This behavior might be due to the form factor describing $D^{0}$ decay to a $K \pi S$-wave system and a bachelor $\bar{K}$. Since no centrifugal barrier is involved, such an effect should be more significant for $S$ waves than for higher spin waves because of the larger overlap between the initial and final state wave functions. However, the inverse momentum of the $K \pi$ system in the $D^{0}$ rest frame increases from 0.27 Fermi at $K \pi$ threshold to 0.48 Fermi at $1.15 \mathrm{GeV} / c^{2}$; therefore any form factor effect would decrease with increasing $K \pi$ mass. If the effect is essentially gone by $1.15 \mathrm{GeV} / c^{2}$, similar mass dependence of amplitude and phase in $D^{0}$ decay and $K \pi$ scattering would be observable at higher mass values, in agreement with Fig. 2. In the present analysis, we make an attempt to distinguish between the two rather different $K \pi$ $S$-wave mass dependences in the region below $\sim 1.15 \mathrm{GeV} / c^{2}$. In each case, we also allow the fit to determine the strength and phase of these amplitudes relative to the $K^{*}(892)^{+}$reference.

We describe the $D^{0}$ decay to a $K^{-} K^{+} S$-wave state by a coupled-channel Breit-Wigner amplitude for the $f_{0}(980)$ and $a_{0}(980)$ resonances, with their respective couplings to $\pi \pi, K \bar{K}$ and $\eta \pi, K \bar{K}$ final states [15],

$$
A_{f_{0}\left[a_{0}\right]}(s)=\frac{M_{D^{0}}^{2}}{M_{0}^{2}-s-i\left(g_{1}^{2} \rho_{\pi \pi[\eta \pi]}+g_{2}^{2} \rho_{K \bar{K}}\right)} .
$$

Here $\rho$ represents Lorentz invariant phase space, $2 p / \sqrt{s}$. For the $f_{0}(980)$, we use the BES [16] parameter values $M_{0}=965 \pm 10 \mathrm{MeV} / c^{2}, g_{1}^{2}=165 \pm 18 \mathrm{MeV}^{2} / c^{4}$, and $g_{2}^{2} / g_{1}^{2}=4.21 \pm 0.33$. For the $a_{0}(980)$, we use the Crystal Barrel [17] values $M_{0}=999 \pm 2 \mathrm{MeV} / c^{2}, \quad g_{1}=$ $324 \pm 15 \mathrm{MeV} / c^{2}$, and $g_{1}^{2} / g_{2}^{2}=1.03 \pm 0.14$. Only the high mass tails of $f_{0}(980)$ and $a_{0}(980)$ are observable, as shown in Fig. 3(a). They are similar, so we try a model for

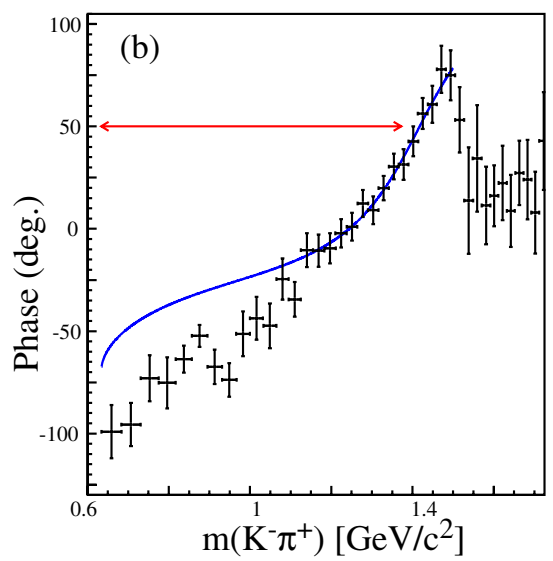

FIG. 2 (color online). LASS (solid line, blue) and E-791 (dots with error bars) $K \pi S$-wave amplitudes (a), in arbitrary units, and phase (b). The double headed arrow (red) indicates the mass range available in the decay $D^{0} \rightarrow K^{-} K^{+} \pi^{0}$. 

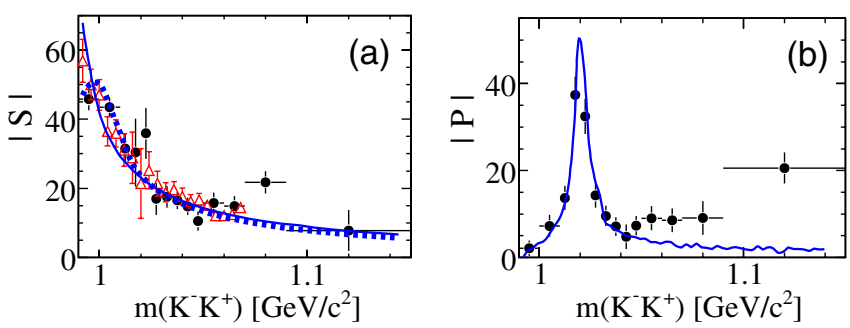

FIG. 3 (color online). The phase-space-corrected $K^{-} K^{+} S$ and $P$-wave amplitudes, $|S|$ and $|P|$, respectively, in arbitrary units, as functions of the invariant mass. (a) Line shapes for $f_{0}(980)$ (solid line, blue) and $a_{0}(980)$ (broken line, blue), derived from Eq. (7). (b) Line shape for $\phi(1020)$ (solid line, blue). In each plot, solid circles with error bars correspond to values obtained from the model-independent analysis for $|S|$ and $|P|$ using Eq. (8). In (a), the open triangles (red) correspond to values obtained from the decay $D^{0} \rightarrow K^{-} K^{+} \bar{K}^{0}$ (see text).

each as a description of the $K^{-} K^{+} S$-wave amplitude. In Fig. 3(b) we show, in the same mass range, the $K^{-} K^{+}$ $P$-wave amplitude parametrized by the $\phi(1020)$ meson.

To fit the Dalitz plot, we try several models incorporating various combinations of intermediate states. In each fit, we include the $K^{*}(892)^{+}$and measure the complex amplitude coefficients of other states relative to it. As a check on the quality of each fit, we compare the number of events observed in bins in the Dalitz plot with the number predicted by the fit. We compute residuals and statistical uncertainties to form a $\chi^{2}$, and take $\chi^{2} / \nu$ (where $\nu$ is the number of bins less the number of variable parameters) as a figure of merit. We also compare the distributions of angular moments (described later) predicted by the fit and actually observed in the data.

The LASS $K \pi S$-wave amplitude gives the best agreement with data and we use it in our nominal fits (see next paragraph). The $K \pi S$-wave modeled by the combination of $\kappa(800)$ (with parameters taken from Ref. [3]), a nonresonant term, and $K_{0}^{*}(1430)$ has a smaller fit probability $\left(\chi^{2}\right.$ probability $\left.<5 \%\right)$. The best fit with this model $\left(\chi^{2}\right.$ probability $13 \%$ ) yields a charged $\kappa$ of mass (870 \pm 30) $\mathrm{MeV} / c^{2}$, and width $(150 \pm 20) \mathrm{MeV} / c^{2}$, significantly different from those reported in Ref. [3] for the neutral state. This does not support the hypothesis that production of a charged, scalar $\kappa$ is being observed. The E-791 amplitude [14] describes the data well, except near threshold ( $\chi^{2}$ probability $23 \%$ ). Though our data favor the LASS parametrization for $\sqrt{s}<1.15 \mathrm{GeV} / c^{2}$, the insensitivity of the fit to small variations in amplitude at these masses does not allow an independent $S$-wave measurement with the present data sample. Therefore, we use the E-791 amplitude to estimate systematic uncertainty in our results.

We find that two different isobar models describe the data well. Both yield almost identical behavior in invariant mass [Figs. 1(b)-1(d)] and angular distribution (Fig. 4). We use LASS amplitude to describe the $K \pi S$-wave amplitudes in both the isobar models (I and II). We summarize the results of the best fits (model I: $\chi^{2} / \nu=702.08 / 714$, probability $61.9 \%$; model II: $\chi^{2} / \nu=718.89 / 717$, probability $47.3 \%$ ) in Table I. We also list the fit fraction for each resonant process $r$, defined as $f_{r} \equiv \int\left|a_{r} A_{r}\right|^{2} d \tau /$ $\int\left|f_{D^{0}}\right|^{2} d \tau$, where $d \tau=d m_{K^{-} \pi^{0}}^{2} d m_{K^{+} \pi^{0}}^{2}$, in Table I. Because of interference among the contributing amplitudes, the $f_{r}$ do not sum to one in general. We find that the $K \pi S$-wave is not in phase with the $P$-wave at threshold as it was in the LASS scattering data. For model I (II), the $S$-wave phase relative to the $K^{*}(892)^{+}$is $\sim 180^{\circ}\left(150^{\circ}\right)$ for the positive charge and $135^{\circ}\left(110^{\circ}\right)$ for the negative charge.

We have also considered the possible contributions from other resonant states such as $K_{2}^{*}(1430), f_{2}(1270)$,
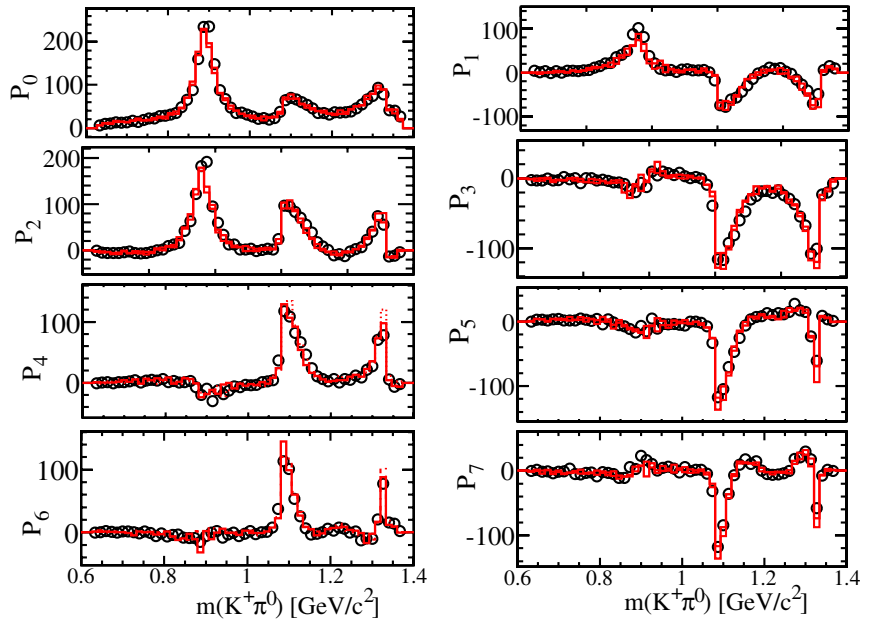

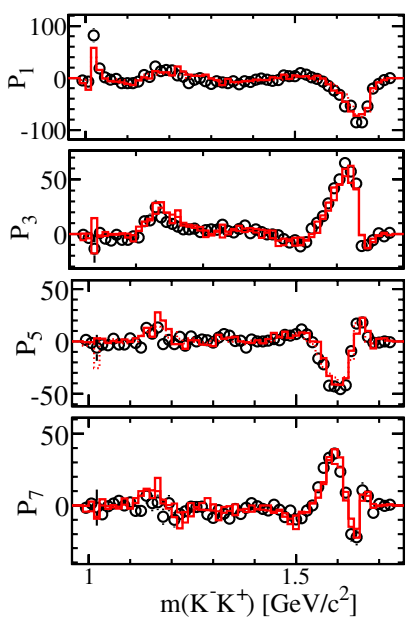

FIG. 4 (color online). The mass dependence of the spherical harmonic moments of $\cos \theta_{H}$ after efficiency corrections and background subtraction: $K^{+} \pi^{0}$ (columns I, II) and $K^{-} K^{+}$(columns III, IV). The circles with error bars are data points and the curves (red) are derived from the fit functions (see text). For the sake of visibility, we do not show error bars on the curves. 
TABLE I. The results obtained from the $D^{0} \rightarrow K^{-} K^{+} \pi^{0}$ Dalitz plot fit. We define amplitude coefficients, $a_{r}$ and $\phi_{r}$, relative to those of the $K^{*}(892)^{+}$. The errors are statistical and systematic, respectively. We show the $a_{0}(980)$ contribution, when it is included in place of the $f_{0}(980)$, in square brackets. We denote the $K \pi S$-wave states here by $K^{ \pm} \pi^{0}(S)$. We use the LASS amplitude to describe the $K \pi S$-wave states in both the isobar models (I and II).

\begin{tabular}{|c|c|c|c|c|c|c|}
\hline State & Amplitude, $a_{r}$ & $\begin{array}{c}\text { Model I } \\
\text { Phase, } \phi_{r}\left({ }^{\circ}\right)\end{array}$ & Fraction, $f_{r}(\%)$ & Amplitude, $a_{r}$ & $\begin{array}{l}\text { Model II } \\
\text { Phase, } \phi_{r}\left(^{\circ}\right)\end{array}$ & Fraction, $f_{r}(\%)$ \\
\hline$K^{*}(892)^{+}$ & 1.0 (fixed) & 0.0 (fixed) & $45.2 \pm 0.8 \pm 0.6$ & 1.0 (fixed) & 0.0 (fixed) & $44.4 \pm 0.8 \pm 0.6$ \\
\hline$K^{*}(1410)^{+}$ & $2.29 \pm 0.37 \pm 0.20$ & $86.7 \pm 12.0 \pm 9.6$ & $3.7 \pm 1.1 \pm 1.1$ & & & \\
\hline$K^{+} \pi^{0}(S)$ & $1.76 \pm 0.36 \pm 0.18$ & $-179.8 \pm 21.3 \pm 12.3$ & $16.3 \pm 3.4 \pm 2.1$ & $3.66 \pm 0.11 \pm 0.09$ & $-148.0 \pm 2.0 \pm 2.8$ & $71.1 \pm 3.7 \pm 1.9$ \\
\hline$\phi(1020)$ & $0.69 \pm 0.01 \pm 0.02$ & $-20.7 \pm 13.6 \pm 9.3$ & $19.3 \pm 0.6 \pm 0.4$ & $0.70 \pm 0.01 \pm 0.02$ & $18.0 \pm 3.7 \pm 3.6$ & $19.4 \pm 0.6 \pm 0.5$ \\
\hline$f_{0}(980)$ & $0.51 \pm 0.07 \pm 0.04$ & $-177.5 \pm 13.7 \pm 8.6$ & $6.7 \pm 1.4 \pm 1.2$ & $0.64 \pm 0.04 \pm 0.03$ & $-60.8 \pm 2.5 \pm 3.0$ & $10.5 \pm 1.1 \pm 1.2$ \\
\hline$\left[a_{0}(980)^{0}\right]$ & {$[0.48 \pm 0.08 \pm 0.04]$} & {$[-154.0 \pm 14.1 \pm 8.6]$} & {$[6.0 \pm 1.8 \pm 1.2]$} & {$[0.68 \pm 0.06 \pm 0.03]$} & {$[-38.5 \pm 4.3 \pm 3.0]$} & {$[11.0 \pm 1.5 \pm 1.2]$} \\
\hline$f_{2}^{\prime}(1525)$ & $1.11 \pm 0.38 \pm 0.28$ & $-18.7 \pm 19.3 \pm 13.6$ & $0.08 \pm 0.04 \pm 0.05$ & & & \\
\hline$K^{*}(892)^{-}$ & $0.601 \pm 0.011 \pm 0.011$ & $-37.0 \pm 1.9 \pm 2.2$ & $16.0 \pm 0.8 \pm 0.6$ & $0.597 \pm 0.013 \pm 0.009$ & $-34.1 \pm 1.9 \pm 2.2$ & $15.9 \pm 0.7 \pm 0.6$ \\
\hline$K^{*}(1410)^{-}$ & $2.63 \pm 0.51 \pm 0.47$ & $-172.0 \pm 6.6 \pm 6.2$ & $4.8 \pm 1.8 \pm 1.2$ & & & \\
\hline$K^{-} \pi^{0}(S)$ & $0.70 \pm 0.27 \pm 0.24$ & $133.2 \pm 22.5 \pm 25.2$ & $2.7 \pm 1.4 \pm 0.8$ & $0.85 \pm 0.09 \pm 0.11$ & $108.4 \pm 7.8 \pm 8.9$ & $3.9 \pm 0.9 \pm 1.0$ \\
\hline
\end{tabular}

$f_{0}(1370)$, and $f_{0}(1510)$. We find that none of them is needed to describe the Dalitz plot; they all provide small contributions and lead to smaller $\chi^{2}$ probabilities.

Angular distributions provide more detailed information on specific features of the amplitudes used in the description of the Dalitz plot. We define the helicity angle $\theta_{H}$ for the decay $D^{0} \rightarrow(r \rightarrow A B) C$ as the angle between the momentum of $A$ in the $A B$ rest frame and the momentum of $A B$ in the $D^{0}$ rest frame. The moments of $\cos \theta_{H}$, defined as the efficiency-corrected and background-subtracted invariant mass distributions of events weighted by spherical harmonic functions, $Y_{l}^{0}\left(\cos \theta_{H}\right)=\sqrt{\frac{2 l+1}{4 \pi}} P_{l}\left(\cos \theta_{H}\right)$, where the $P_{l}$ are Legendre polynomials of order $l$, are shown in Fig. 4 for the $K^{+} \pi^{0}$ and $K^{-} K^{+}$channels, for $l=0-7$. The $K^{-} \pi^{0}$ moments are similar to those for $K^{+} \pi^{0}$.

The mass dependent $K^{-} K^{+} S$ - and $P$-wave complex amplitudes can also be obtained directly from our data in a model-independent way in a limited mass range around $1 \mathrm{GeV} / c^{2}$. In a region of the Dalitz plot where $S$ and $P$ waves in a single channel dominate, their amplitudes are given by the following Legendre polynomial moments,

$$
\begin{gathered}
P_{0}=\frac{|S|^{2}+|P|^{2}}{\sqrt{2}}, \quad P_{1}=\sqrt{2}|S||P| \cos \theta_{S P}, \\
P_{2}=\sqrt{\frac{2}{5}|P|^{2}}
\end{gathered}
$$

using $\int_{-1}^{1} P_{l} P_{m} d\left(\cos \theta_{H}\right)=\delta_{l m}$. Here $|S|$ and $|P|$ are, respectively, the magnitudes of the $S$ - and $P$-wave amplitudes, and $\theta_{S P}=\theta_{S}-\theta_{P}$ is the relative phase between them. We use these relations to evaluate $|S|$ and $|P|$, shown in Fig. 3, for the $K^{-} K^{+}$channel in the mass range $m_{K^{-} K^{+}}<1.15 \mathrm{GeV} / c^{2}$. The measured values of $|S|$ agree well with those obtained in the analysis of the decay $D^{0} \rightarrow$ $K^{-} K^{+} \bar{K}^{0}$ [18]. They also agree well with either the $f_{0}(980)$ or the $a_{0}(980)$ line shape. The measured values of $|P|$ are consistent with a Breit-Wigner line shape for $\phi(1020)$. Results for $\cos \theta_{S P}$ and $\theta_{S P}$ are shown in
Figs. 5(a) and 5(b). A twofold ambiguity in the sign of $\theta_{S P}$ exists, as shown in Fig. 5(b). It is, however, straightforward to choose the physical solution. In this region, the $\phi(1020)$ meson ( $P$-wave) has a very rapidly rising phase, while we expect the $S$-wave phase to be relatively slowly varying. Thus, the upper solution, in which $\theta_{S}-\theta_{P}$ is rapidly falling, is the physical solution. We take the Breit-Wigner phase of $\phi(1020)$, shown in Fig. 5(c), to be a good model for $\theta_{P}$ and obtain $\theta_{S}$, as plotted in Fig. 5(d).
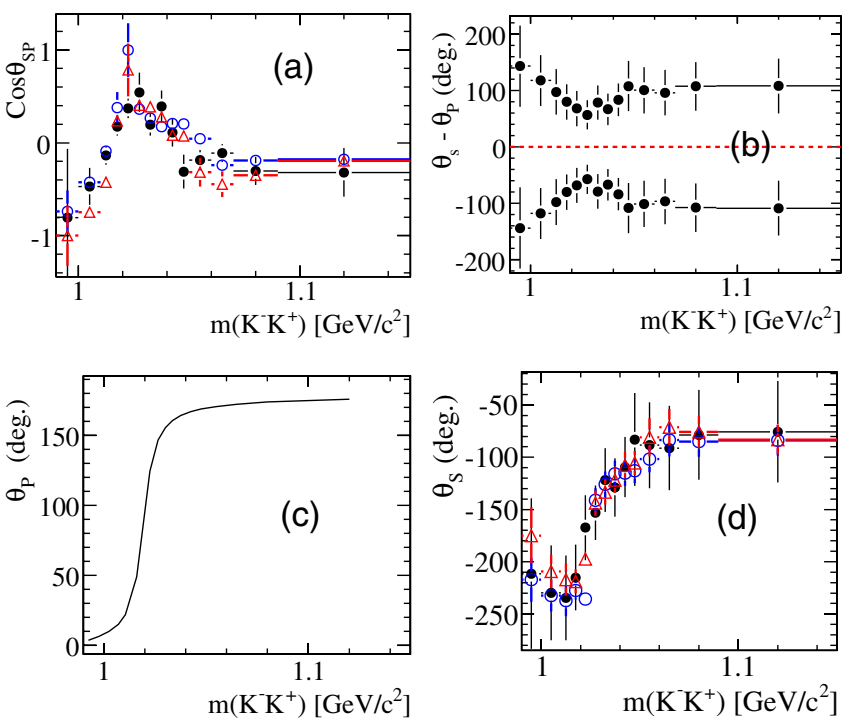

FIG. 5 (color online). Results of the partial-wave analysis of the $K^{-} K^{+}$system using Eq. (8) described in the text. (a) Cosine of the relative phase $\theta_{S P}=\theta_{S}-\theta_{P}$, (b) two solutions for $\theta_{S P}$, (c) the $P$-wave phase taken from Eqs. (3) and (4) for the $\phi(1020)$ meson, and (d) the $S$-wave phase derived from the upper solution in (b). Solid bullets are data points, and open circles (blue) and open triangles (red) correspond, respectively, to isobar models I and II. The number of simulated events used for the two models is 10 times larger than data. Errors for quantities from the isobar models arise from Monte Carlo statistical limitations, and differ from errors derived from Eq. (8). 
These results show little variation in the $S$-wave phase up to about $1.02-1.03 \mathrm{GeV} / c^{2}$, then a rapid rise above that. Also, in Fig. 3(b), we observe that $|P|$ follows the $\phi(1020)$ curve well up to about the same mass, with a significant deviation above that. The behavior observed matches well to that obtained from the isobar models-I or II. No distinction between them appears possible from this analysis. The partial-wave analysis described above is valid, in the absence of higher spin states, only if no interference occurs from the crossing $K \pi$ channels. The behavior observed in both $S$ and $P$ waves above $\sim 1.03 \mathrm{GeV} / c^{2}$ can, therefore, be attributed to high mass tails of the $K^{*}(892)$ and low mass tails of possible higher $K^{*}$ resonances.

Systematic uncertainties in quantities in Table I arise from experimental effects, and also from uncertainties in the nature of the models used to describe the data. We determine these separately and add them in quadrature. In both cases, we assign the maximum deviation in the observed quantities (i.e., $a_{r}, \phi_{r}$, and $f_{r}$ ) from the central value as a systematic uncertainty, taking correlations among fit parameters into account. We characterize the uncertainties due to $K \pi S$-wave amplitudes and resonance mass-width values as model dependent. We estimate them conservatively taking symmetric errors from the spread in results when either the LASS amplitude is replaced by the E-791 amplitude, or the resonance parameters are changed by 1 standard deviation $(\sigma)$. Similarly, we estimate the experimental uncertainty from the variation in results when either the signal efficiency parameters are varied by $1 \sigma$, or the background shape is taken from simulation instead of the data sideband, or the ratio of particle-identification rates in data and simulation is varied by $1 \sigma$. Model and experimental systematics contribute almost equally to the total uncertainty. As a consistency check, we analyze disjoint data samples, in bins of reconstructed $D^{0}$ mass and laboratory momentum, and find consistent results.

Neglecting $C P$ violation, the strong phase difference, $\delta_{D}$, between the $\bar{D}^{0}$ and $D^{0}$ decays to the $K^{*}(892)^{+} K^{-}$ state and their amplitude ratio, $r_{D}$, are given by

$$
r_{D} e^{i \delta_{D}}=\frac{a_{D^{0} \rightarrow K^{*-} K^{+}}}{a_{D^{0} \rightarrow K^{*+} K^{-}}} e^{i\left(\delta_{K^{*-} K^{+}}-\delta_{K^{*+} K^{-}}\right)} .
$$

Combining the results of models I and II, we find $\delta_{D}=$ $-35.5^{\circ} \pm 1.9^{\circ}($ stat $) \pm 2.2^{\circ}$ (syst) and $r_{D}=0.599 \pm$ 0.013 (stat) \pm 0.011 (syst). These results are consistent with the previous measurements [19], $\delta_{D}=-28^{\circ} \pm$ $8^{\circ}($ stat $) \pm 11^{\circ}($ syst $) \quad$ and $\quad r_{D}=0.52 \pm 0.05($ stat $) \pm$ 0.04 (syst).

In conclusion, we have studied the amplitude structure of the decay $D^{0} \rightarrow K^{-} K^{+} \pi^{0}$, and measured $\delta_{D}$ and $r_{D}$. We find that two isobar models give excellent descriptions of the data. Both models include significant contributions from $K^{*}(892)$, and each indicates that $D^{0} \rightarrow K^{*+} K^{-}$dominates over $D^{0} \rightarrow K^{*-} K^{+}$. This suggests that, in tree-level diagrams, the form factor for $D^{0}$ coupling to $K^{*-}$ is suppressed compared to the corresponding $K^{-}$coupling. While the measured fit fraction for $D^{0} \rightarrow K^{*+} K^{-}$agrees well with a phenomenological prediction [20] based on a large SU(3) symmetry breaking, the corresponding results for $D^{0} \rightarrow K^{*-} K^{+}$and the color-suppressed $D^{0} \rightarrow \phi \pi^{0}$ decays differ significantly from the predicted values. It appears from Table I that the $K^{+} \pi^{0} S$-wave amplitude can absorb any $K^{*}(1410)$ and $f_{2}^{\prime}(1525)$ if those are not in the model. The other components are quite well established, independent of the model. The $K \pi S$-wave amplitude is consistent with that from the LASS analysis, throughout the available mass range. We cannot, however, completely exclude the behavior at masses below $\sim 1.15 \mathrm{GeV} / c^{2}$ observed in the decay $D^{+} \rightarrow K^{-} \pi^{+} \pi^{+}$ [3,14]. The $K^{-} K^{+} S$-wave amplitude, parametrized as either $f_{0}(980)$ or $a_{0}(980)^{0}$, is required in both isobar models. No higher mass $f_{0}$ states are found to contribute significantly. In a limited mass range, from threshold up to $1.02 \mathrm{GeV} / c^{2}$, we measure this amplitude using a modelindependent partial-wave analysis. Agreement with similar measurements from $D^{0} \rightarrow K^{-} K^{+} \bar{K}^{0}$ decay [18], and with the isobar models considered here, is excellent.

We are grateful for the excellent luminosity and machine conditions provided by our PEP-II colleagues, and for the substantial dedicated effort from the computing organizations that support $B A B A R$. The collaborating institutions wish to thank SLAC for its support and kind hospitality. This work is supported by DOE and NSF (USA), NSERC (Canada), CEA and CNRS-IN2P3 (France), BMBF and DFG (Germany), INFN (Italy), FOM (The Netherlands), NFR (Norway), MIST (Russia), MEC (Spain), and PPARC (United Kingdom). Individuals have received support from the Marie Curie EIF (European Union) and the A. P. Sloan Foundation.
[1] J. L. Rosner, Phys. Rev. D 74, 076006 (2006); M. R. Pennington, Int. J. Mod. Phys. A 21, 5503 (2006); D. V. Bugg, Phys. Lett. B 632, 471 (2006).

[2] Reference to the charge-conjugate decay is implied throughout. The initial state referred to is $D^{0}$, not $\bar{D}^{0}$.

[3] E. M. Aitala et al. (E-791 Collaboration), Phys. Rev. Lett.
89, 121801 (2002).

[4] A. Giri, Y. Grossman, A. Soffer, and J. Zupan, Phys. Rev. D 68, 054018 (2003).

[5] B. Aubert et al. (BABAR Collaboration), arXiv:hep-ex/ 0703037.

[6] Y. Grossman, A. L. Kagan, and Y. Nir, Phys. Rev. D 75, 
036008 (2007), and references therein.

[7] B. Aubert et al. (BABAR Collaboration), Nucl. Instrum. Methods Phys. Res., Sect. A 479, 1 (2002).

[8] B. Aubert et al. (BABAR Collaboration), Phys. Rev. D 74, 091102 (2006).

[9] S. J. Lindenbaum and R. M. Sternheimer, Phys. Rev. 105, 1874 (1957); M. G. Olsson and G. V. Yodh, Phys. Rev. 145, 1309 (1966); D. J. Herndon, P. Söding, and R. J. Cashmore, Phys. Rev. D 11, 3165 (1975).

[10] W.-M. Yao et al. (PDG Collaboration), J. Phys. G 33, 1 (2006).

[11] J.M. Blatt and W.F. Weisskopf, Theoretical Nuclear Physics (John Wiley \& Sons, New York, 1952).

[12] H. Albrecht et al. (ARGUS Collaboration), Phys. Lett. B 308, 435 (1993).
[13] D. Aston et al. (LASS Collaboration), Nucl. Phys. B296, 493 (1988); W. M. Dunwoodie (private communication).

[14] E. M. Aitala et al. (E-791), Phys. Rev. D 73, 032004 (2006); B. T. Meadows (private communication).

[15] S. M. Flatte, Phys. Lett. 38B, 232 (1972).

[16] M. Ablikim et al. (BES Collaboration), Phys. Lett. B 607, 243 (2005).

[17] A. Abele et al. (Crystal Barrel Collaboration), Phys. Rev. D 57, 3860 (1998).

[18] B. Aubert et al. (BABAR Collaboration), Phys. Rev. D 72, 052008 (2005).

[19] C. Cawlfield et al. (CLEO Collaboration), Phys. Rev. D 74, 031108 (2006).

[20] F. Buccella et al., Phys. Rev. D 51, 3478 (1995). 